\title{
THE DIRICHLET PROBLEM FOR HARMONIC MAPS FROM A SURFACE WITH BOUNDARY ONTO A 2-SPHERE WITH NONCONSTANT BOUNDARY VALUES
}

\author{
JÜRGEN JOST
}

\section{Discussion of the main result}

Let $\Sigma_{1}$ and $\Sigma_{2}$ be two compact two-dimensional Riemannian manifolds, $\partial \Sigma_{2}=\varnothing$. In [8] and [9], Lemaire showed that if $\pi_{2}\left(\Sigma_{2}\right)=0$ every map $\varphi$ from $\Sigma_{1}$ to $\Sigma_{2}$ is homotopic to a harmonic map $u$ which minimizes energy in its homotopy class and satisfies $u\left|\partial \Sigma_{1}=\varphi\right| \partial \Sigma_{1}$ in case $\partial \Sigma_{1} \neq \varnothing$. (If $\partial \Sigma_{1}=\varnothing$, this was proved independently by Sacks and Uhlenbeck [10]; cf. also [11].) If $\pi_{2}\left(\Sigma_{2}\right) \neq 0$, then such an existence result is no more true, however. Lemaire showed in [8] that the minimum of energy is not attained among maps of degree 1 from a surface of positive genus onto the two-dimensional sphere $S^{2}$, and hence there does not exist an energy minimizing harmonic map in those homotopy classes. Actually, Eells and Wood [3] proved that there is no harmonic map at all from the torus onto $S^{2}$ of degree 1. Furthermore, concerning the boundary value problem, Lemaire also demonstrated in [8] that any harmonic map from the unit disc onto $S^{2}$ with constant boundary values has to be constant itself.

Hence, in this case the Dirichlet problem cannot be solved in any nontrivial homotopy class. Lemaire's proof consists in showing that such a harmonic map necessarily has to be conformal and therefore constant, since there exists no surjective conformal map from the disc onto $S^{2}$. In particular, this implies that the limit of an energy minimizing sequence has to fall out of a nontrivial homotopy class and has to become trivial. Lemaire's argument, however, raises the question what happens if the prescribed boundary values are nonconstant (and, e.g., separate $S^{2}$ into two different regions which are conformally equivalent to the disc) and more precisely, whether one can find at least two

Received October 18, 1983. Research supported by SFB 72 at the University of Bonn. 
homotopically different harmonic maps which minimize energy in their respective homotopy classes.

The purpose of the present note is to provide an affirmative answer to this question. Actually we shall prove the following more general result.

Theorem. Suppose $\Sigma_{1}$ is a compact two-dimensional Riemannian manifold with nonempty boundary $\partial \Sigma_{1}$, and $\Sigma_{2}$ is a Riemannian manifold homeomorphic to $S^{2}$ (the standard 2-sphere), and $\psi: \partial \Sigma_{1} \rightarrow \Sigma_{2}$ is a continuous map, not mapping $\partial \Sigma_{1}$ onto a single point and admitting a continuous extension to a map from $\Sigma_{1}$ to $\Sigma_{2}$ with finite energy. Then there are at least two homotopically different harmonic maps $u$ : $\Sigma_{1} \rightarrow \Sigma_{2}$ with $u \mid \partial \Sigma_{1}=\psi$, which minimize energy in their respective homotopy classes.

Again, it might be asked whether this result is optimal or whether the minimum of energy is attained in every homotopy class of maps from the disc, e.g., onto $S^{2}$ with nonconstant boundary values. This is not the case, however, as the following easy modification of Lemaire's argument $[8$, p. 65f] shows where again the nonexistence of a conformal map will imply the nonexistence of an energy minimizing map.

Let $D$ be the unit disc in the complex plane, and $k: D \rightarrow S^{2}$ be a conformal map mapping $D$ onto the upper hemisphere and $\partial D$ onto the equator. Furthermore, suppose that $k$ is equivariant with respect to the rotations of $D$ and $S^{2}$ (the latter ones leaving the north and south pole of $S^{2}$ fixed). We choose the orientation on $S^{2}$ in such a way that the Jacobian of $k$ is positive.

Let $D(0, r)$ be the plane disc with center 0 and radius $r$ (i.e. $D=D(0,1)$ ). Let $h_{r}$ be a map from $D(0, r)$ onto $S^{2}$ which maps $\partial D(0, r)$ onto the north pole, is injective in the interior of $D(0, r)$ and has a positive Jacobian there, and is $\varepsilon$-conformal. We introduce polar coordinates $(\rho, \varphi)$ on $D$ and define for $0<r<1$ the mapping $k_{r}$ by

$$
k_{r}(\rho, \varphi)= \begin{cases}k\left(\frac{1}{1-r} \rho+\frac{r}{r-1}, \varphi\right) & \text { if } r \leqslant \rho \leqslant 1, \\ h_{r}(\rho, \varphi) & \text { if } 0 \leqslant \rho \leqslant r .\end{cases}
$$

Using the $\varepsilon$-conformality theorem it is easy to see that the energy of $k_{r}$ can be made arbitrarily close to $6 \pi$ if we choose $r>0$ sufficiently small.

On the other hand, $6 \pi$ is just the area of the image of $k_{r}$, counted with multiplicity. Hence, if there is an energy minimizing map homotopic to $k_{r}$, its energy has to be equal to the area of the image, and it therefore has to be conformal. Since the boundary values are equivariant, this conformal map itself has to be equivariant (otherwise there would exist infinitely many homotopic conformal maps with the same boundary values which is not possible). This, however, implies that it would have to collapse a circle in $D$ to 
a point which is not possible for a conformal map. Hence there is no energy minimizing map homotopic to $k_{r}$.

By letting $h_{r}$ cover $S^{2}$ more than once, we obtain other classes without energy minimizing maps by a similar argument. If $h_{r}$, however, has degree -1 , then $k_{r}$ is homotopic to a map of $D$ onto the lower hemisphere and hence homotopic to an energy minimizing map. Hence, in this example, there are precisely two homotopy classes which contain energy minimizing maps.

Lemaire's argument also indicates that what can go wrong with a minimizing sequence is the "bubbling off" of a sphere with some multiplicity. (That this is indeed the only way a limit of a minimizing sequence can leave its homotopy class was shown by Sacks and Uhlenbeck [10].)

Our proof will use some ideas introduced in our previous paper [6]. We shall show with the help of a maximum principle and a careful replacement argument that the minimum of energy is attained in two different homotopy classes and hence in those classes no sphere can bubble off. These classes are related to each other by attaching a sphere with multiplicity -1 . Our replacement argument was inspired by Wente's paper [14]. One should note that the fact that if a sphere bubbles off the energy has to drop at least by the area of the image, was also used in the recent solution of the Rellich conjecture by Brézis and Coron [1] (an independent, though somewhat weaker solution was provided by Struwe [13] and Steffen [12]).

I am grateful to A. Baldes and M. Struwe for drawing my attention upon this problem. After I had finished the first version of my paper, J. M. Coron informed me that a similar result had been obtained by Brézis and himself. Their work will appear in [2]. I thank him for several useful comments on my paper. In particular, the discussion with him led me to discover an error of mine concerning the topological setting of the problem and to work out the remark following the statement of the result. A proof of this latter fact by direct calculation will also appear in [2].

For a detailed account of the theory of harmonic maps between surfaces the reader can consult [7].

\section{Some lemmata}

An essential tool in our proof of the theorem will be the following maximum principle.

Lemma 1. Suppose $B(p, s):=\{q \in N: d(p, q) \leqslant s\}$ is a geodesic ball in a Riemannian manifold $N$ with distance function $d(\cdot, \cdot)$, and

$$
s \leqslant \frac{1}{2} \min (i(p), \pi / 2 \kappa),
$$


where $i(p)$ is the injectivity radius of $p$, and $\kappa^{2}$ is an upper bound for the sectional curvature of $N$.

If $h: \Omega \rightarrow N, \Omega$ being a domain on some Riemannian manifold, is energy minimizing among maps which are homotopic to some $g: \Omega \rightarrow B(p, s)$, and if $h(\partial \Omega) \subset B(p, s)$, then also $h(\Omega) \subset B(p, s)$.

For a proof, cf. [6].

Furthermore, we shall make use of the following result of Hildebrandt, Kaul and Widman [5] (cf. [6] for an easy proof).

Lemma 2. Suppose $\Omega$ is a domain on some surface $\Sigma_{1}, \partial \Omega \neq 0$, and $B(p, M)$ is a disc on another surface $\Sigma_{2}$, with $M<\pi / 2 \kappa$, where $\kappa^{2}$ is an upper bound for the Gauss curvature on $B(p, M)$, and $\psi: \partial \Omega \rightarrow B(p, M)$ is continuous and admits an extension $\bar{\psi}: \Omega \rightarrow B(p, M)$ with finite energy $E(\bar{\psi})$.

Then there exists a harmonic map $h: \Omega \rightarrow B(p, M)$ with $h \mid \partial \Omega=\psi$, and $h$ minimizes the energy with respect to these boundary values. Conversely, each such energy minimizing map is harmonic. The modulus of continuity of $h$ can be estimated in terms of a lower bound of the Gauss curvature of $\Sigma_{1}$, the injectivity radius $i\left(\Sigma_{1}\right), M, \kappa, E(\bar{\psi})$, and the modulus of continuity of $\psi$.

Finally, we shall use the following result due to Lebesgue and Courant (cf. [7, Lemma 3.1]).

Lemma 3. Suppose $u: \Omega \rightarrow \Sigma_{2}, \Omega$ being a domain of some surface $\Sigma_{1}$, is a map with finite energy $E(u), x_{0} \in \bar{\Omega},-\lambda^{2}$ is a lower curvature bound on $\bar{\Omega}$, and

$$
\delta<\min \left(1, i\left(\Sigma_{1}\right), 1 / \lambda^{2}\right) .
$$

Then there exists some $r \in(\delta, \sqrt{\delta})$ with

$$
d\left(u\left(x_{1}\right), u\left(x_{2}\right)\right) \leqslant 2 \pi E(u)^{1 / 2}(\log 1 / \delta)^{-1 / 2}
$$

for all $x_{1}, x_{2} \in \bar{\Omega}$ with $d\left(x_{i}, x_{0}\right)=r(i=1,2)$.

\section{Proof of the theorem}

We put $s=\frac{1}{2} \min \left(i\left(\Sigma_{2}\right), \pi / 2 \kappa\right)$, where $\kappa^{2}$ is an upper curvature bound on $\Sigma_{2}$ and $i\left(\Sigma_{2}\right)$ is the injectivity radius of $\Sigma_{2}$.

Let $\delta_{0} \leqslant \min \left(1, i\left(\Sigma_{1}\right)^{2}, 1 / \lambda^{2}\right)\left(-\lambda^{2}\right.$ being a lower bound for the curvature of $\Sigma_{1}$ ) satisfy

$$
2 \pi \cdot E(\varphi)^{1 / 2}\left(\log 1 / \delta_{0}\right)^{-1 / 2} \leqslant s / 2
$$

where $E(\varphi)$ is the energy of $\varphi$, and

$$
d\left(x_{1}, x_{2}\right) \leqslant \sqrt{\delta_{0}} \Rightarrow d\left(\varphi\left(x_{1}\right), \varphi\left(x_{2}\right)\right) \leqslant s / 2 \text { for } x_{1}, x_{2} \in \partial \Sigma_{1} .
$$


Let $0<\delta \leqslant \delta_{0}$. There exists a finite number of points $x_{i} \in \Sigma_{1}, i=1, \cdots m=$ $m(\delta)$, for which the discs $B\left(x_{i}, \delta / 2\right)$ cover $\Sigma_{1}$. We let $u_{n}$ be a continuous energy minimizing sequence in $[\varphi], E\left(u_{n}\right) \leqslant E(\varphi)$ w.l.o.g. for all $n$.

Applying Lemma 3 and using (1) and (2), for every $n$, we can find $r_{n, 1}$, $\delta<r_{n, 1}<\sqrt{\delta}$, and $p_{n, 1} \in \Sigma_{2}$ with the property that

$$
u_{n}\left(\partial B\left(x_{1}, r_{n, 1}\right)\right) \subset B\left(p_{n, 1}, s\right) .
$$

(Here, we have defined $B(x, r)=\left\{y \in \Sigma_{1}: d(x, y) \leqslant r\right\}$ and thus $\partial B(x, r)=$ $\partial\left(B(x, r) \cap \Sigma_{1}\right)$ near the boundary. $)$

We now have two possibilities: either

(1) There exists some $\delta, 0<\delta \leqslant \delta_{0}$, with the property that for any $x \in \Sigma_{1}$, every sufficiently large $n$, and some $r$ with $\delta<r \leqslant \sqrt{\delta}$ and with $u_{n}(\partial B(x, r))$ $\subset B(p, s)$ for some $p \in \Sigma_{2}, u_{n} \mid B(x, r)$ is homotopic to the solution of the Dirichlet problem

$$
\begin{aligned}
g: B(x, r) \rightarrow B(p, s) \text { harmonic and energy minimizing, } \\
g\left|\partial B(x, r)=u_{n}\right| \partial B(x, r)
\end{aligned}
$$

(the existence of $g$ is ensured by Lemma 2; $g$ is actually unique, but this is not needed in the following constructions); or

(2) Possibly choosing a subsequence of the $u_{n}$, we can find a sequence of points $x_{n} \in \Sigma_{1}$, and radii $r_{n}>0, x_{n} \rightarrow x_{0} \in \Sigma_{1}, r_{n} \rightarrow 0$, with $u_{n}\left(\partial B\left(x_{n}, r_{n}\right)\right) \subset$ $B\left(p_{n}, \varepsilon_{n}\right)$ for some $p_{n} \in \Sigma_{2}, p_{n} \rightarrow p \in \Sigma_{2}, \varepsilon_{n} \rightarrow 0$ (using Lemma 3), but for which $u_{n} \mid B\left(x_{n}, r_{n}\right)$ is not homotopic to the solution of the Dirichlet problem (4).

In case (1), we replace $u_{n}$ on $B\left(x_{1}, r_{n, 1}\right)$ by the solution of the Dirichlet problem (4) for $x=x_{1}$ and $r=r_{n, 1}$. We can assume $r_{n, 1} \rightarrow r_{1}$ and $p_{n, 1} \rightarrow p_{1} \in$ $\Sigma_{2}$, and, using the interior modulus of continuity estimates for the solution of (4), that the replaced maps, denoted by $u_{n}^{1}$, converge uniformly on $B\left(x_{1}, \delta-\eta\right)$, for any $0<\eta<\delta$. By Lemma 1

$$
E\left(u_{n}^{1}\right) \leqslant E\left(u_{n}\right) \text {. }
$$

By the same argument as above, we then find radii $r_{n, 2}, \delta<r_{n, 2}<\sqrt{\delta}$, with

$$
u_{n}^{1}\left(\partial B\left(x_{2}, r_{n, 2}\right)\right) \subset B\left(p_{n, 2}, s\right)
$$

for points $p_{n, 2} \in \Sigma_{2}$.

Again, we replace $u_{n}^{1}$ on $B\left(x_{2}, r_{n, 2}\right)$ by the solution of the Dirichlet problem (4) for $x=x_{2}$ and $r=r_{n, 2}$. We denote the new maps by $u_{n}^{2}$. Again, w.l.o.g., $r_{n, 2} \rightarrow r_{2}$. If we take into consideration that, by the first replacement step, $u_{n}^{1}$ in particular converges uniformly on $B\left(x_{2}, r_{2}\right) \cap B\left(x_{1}, \delta-\eta / 2\right)$, if $0<\eta<\delta$, we see that the boundary values for our second replacement step converge 
uniformly on $\partial B\left(x_{2}, r_{n, 2}\right) \cap B\left(x_{1}, \delta-\eta / 2\right)$. Using the estimates for the modulus of continuity for the solution of (4) at these boundary points (cf. Lemma 2 ), we can assume that the maps $u_{n}^{2}$ converge uniformly on $B\left(x_{1}, \delta-\eta\right) \cup$ $B\left(x_{2}, \delta-\eta\right)$, if $0<\eta<\delta$.

Furthermore, by Lemma 1 again and (5),

$$
E\left(u_{n}^{2}\right) \leqslant E\left(u_{n}^{1}\right) \leqslant E\left(u_{n}\right) .
$$

In this way, we repeat the replacement argument, until we get a sequence $u_{n}^{m}=: v_{n}$ with

$$
E\left(v_{n}\right) \leqslant E\left(u_{n}\right),
$$

which converges uniformly on all balls $B\left(x_{i}, \delta / 2\right), i=1, \cdots m$, and hence on all of $\Sigma_{1}$, since these balls cover $\Sigma_{1}$.

We denote the limit of the $v_{n}$ by $u$. By uniform convergence, $u_{n}$ is homotopic to $\varphi$. Since $E\left(v_{n}\right) \leqslant E(\varphi)$ by (6), we can assume that the $v_{n}$ converge also weakly in $H_{2}^{1}$ to $u$, and by lower semicontinuity of the energy w.r.t. weak $H_{2}^{1}$ convergence and since the $v_{n}$ are a minimizing sequence by (6), $u$ minimizes energy in its homotopy class.

In particular, $u$ minimizes energy when restricted to small balls, and hence it is harmonic and regular by Lemma 2.

We now investigate what happens in case (2). W.l.o.g., $B\left(p_{n}, \varepsilon_{n}\right) \subset B\left(p, 2 \varepsilon_{n}\right)$, and $\varepsilon_{n} \leqslant s / 2$ for all $n$, and thus the solution $g$ of (4) for $x=x_{n}, r=r_{n}$ is contained in $B\left(p, 2 \varepsilon_{n}\right)$ by Lemma 1 . Since $u_{n} \mid B\left(x_{n}, r_{n}\right)$ is not homotopic to $g$, it has to cover $\Sigma_{2} \backslash B\left(p, 2 \varepsilon_{n}\right)$. If we define

$$
\tilde{u}_{n}= \begin{cases}u_{n} & \text { on } \Sigma_{1} \backslash B\left(x_{n}, r_{n}\right), \\ g & \text { on } B\left(x_{n}, r_{n}\right)\end{cases}
$$

then we see that

$$
\begin{aligned}
\lim E\left(u_{n}\right) & \geqslant \lim E\left(u_{n} \mid \Sigma_{1} \backslash B\left(x_{n}, r_{n}\right)\right)+\lim E\left(u_{n} \mid B\left(x_{n}, r_{n}\right)\right) \\
& \geqslant \lim E\left(\tilde{u}_{n}\right)+\operatorname{Area}\left(\Sigma_{2}\right),
\end{aligned}
$$

since $E(g) \rightarrow 0$ as $n \rightarrow \infty$, because

$$
\int_{0}^{2 \pi}\left|\frac{\partial g}{\partial \theta}\left(r_{n}, \theta\right)\right|^{2} d \theta \rightarrow 0
$$

as $n \rightarrow \infty$, if we choose $r_{n}$ as in the proof of Lemma 3. (Furthermore, it is elementary that $E(v \mid B) \geqslant \operatorname{Area}(v(B))$, and equality holds if and only if $v$ is conformal.) We now define

$$
E_{\alpha}:=\inf \{E(v): v \in \alpha\}
$$


for a homotopy class $\alpha$ of maps with $v \mid \partial \Sigma_{1}=\psi$, and $E:=\min _{\alpha} E_{\alpha}$. We first show the existence of a minimizing harmonic map in any homotopy class $\alpha$ with

$$
E_{\alpha}<E+\operatorname{Area}\left(\Sigma_{2}\right)
$$

We choose a minimizing sequence $u_{n}$ in $\alpha$ with

$$
E\left(u_{n}\right)<E+\operatorname{Area}\left(\Sigma_{2}\right) \text {. }
$$

Assuming that case (2) holds, we define $\tilde{u}_{n}$ as above. Since clearly $E\left(\tilde{u}_{n}\right) \geqslant E$, this would contradict (7), however. Therefore, as shown above, we obtain an energy minimizing harmonic map in $\alpha$.

Now let $\tilde{\alpha}$ be a homotopy class with $E_{\tilde{\alpha}}=E$, and let $\tilde{u}$ be an energy minimizing map in $\tilde{\alpha}$, i.e. $E(\tilde{u})=E$. We want to construct a map $v$ in some homotopy class $\alpha \neq \tilde{\alpha}$ with

$$
E(v)<E(\tilde{u})+\operatorname{Area}\left(\Sigma_{2}\right) .
$$

Then the arguments above show that we can find a harmonic map of minimal energy in $\alpha$. In order to complete the proof, it thus only remains to construct $v$.

The metric on $\Sigma_{2}$ is conformally equivalent to the standard metric on $S^{2}$, and thus, we can use $S^{2}$ as a parameter domain for the image.

Since $\psi$ is not a constant map, also $\tilde{u}$ is not a constant map, and hence we can find a point $x_{0}$ in the interior of $\Sigma_{1}$ for which $d \tilde{u}\left(x_{0}\right) \neq 0$. Rotating $S^{2}$, we can assume that $\tilde{u}\left(x_{0}\right)$ is the south pole $p_{0}$. We introduce local coordinates on the image by stereographic projection $\pi: S^{2} \rightarrow \mathrm{C}$ from the south pole $p_{0}$. $d \pi\left(p_{0}\right)$ then is the identity map up to a conformal factor. By Taylor's theorem, $\pi \circ \tilde{u} \mid \partial B\left(x_{0}, \varepsilon\right)$ is a linear map up to an error of order $O\left(\varepsilon^{2}\right)$, i.e.

$$
\left|\pi \circ \tilde{u}(x)-d(\pi \circ \tilde{u})\left(x_{0}\right)\left(x-x_{0}\right)\right|=O\left(\varepsilon^{2}\right)
$$

for $x \in \partial B\left(x_{0}, \varepsilon\right)$.

We now look at conformal maps of the form

$$
w=a z+\frac{b}{z}, \quad a, b \in \mathbf{C}, a=a_{1}+i a_{2}, b=b_{1}+i b_{2} .
$$

The restriction of such a map to a circle $\rho(\cos \theta+i \sin \theta)$ in $\mathbf{C}$ is given by

$$
\begin{aligned}
& u=\left(a_{1} \rho+b_{1} / \rho\right) \cos \theta+\left(b_{2} / \rho-a_{2} \rho\right) \sin \theta, \\
& v=\left(a_{2} \rho+b_{2} / \rho\right) \cos \theta+\left(a_{1} \rho-b_{1} / \rho\right) \sin \theta,
\end{aligned}
$$

where $w=u+i v$.

Therefore, we can choose $a$ and $b$ in such a way that $w$ restricted to this circle coincides with any prescribed nonconstant linear map. This map is nonsingular if

$$
\rho^{4} \neq\left(b_{1}^{2}+b_{2}^{2}\right) /\left(a_{1}^{2}+a_{2}^{2}\right)
$$


W.1.o.g. $\rho^{4} \leqslant\left(b_{1}^{2}+b_{2}^{2}\right) /\left(a_{1}^{2}+a_{2}^{2}\right)$ (otherwise we perform an inversion at the unit circle). Hence $w$ can be extended as a conformal map from the interior of the circle $\rho(\cos \theta+i \sin \theta)$ onto the exterior of its image. We are now in a position to define $v$. On $\Sigma_{1} \backslash B\left(x_{0}, \varepsilon\right)$ we set $v=\tilde{u}$. On $B\left(x_{0}, \varepsilon-\varepsilon^{2}\right)$ we choose a conformal $w$ as above which coincides on the boundary with the linear map $d(\pi \circ \tilde{u})\left(x_{0}\right) /(1-\varepsilon)$, and put

$$
v=\pi^{-1} \circ w .
$$

On $B\left(x_{0}, \varepsilon\right) \backslash B\left(x_{0}, \varepsilon-\varepsilon^{2}\right)$ we interpolate in the following way. We introduce polar coordinates $r, \phi$ and define

$$
\begin{gathered}
f(\phi):=(\pi \circ \tilde{u})(\varepsilon, \phi), \\
g(\phi):=d(\pi \circ \tilde{u})\left(x_{0}\right)(\varepsilon, \phi)=\frac{1}{1-\varepsilon} d(\pi \circ \tilde{u})\left(x_{0}\right)\left(\varepsilon-\varepsilon^{2}, \phi\right), \\
\quad t(r, \phi):=(f(\phi)-g(\phi)) \frac{r}{\varepsilon^{2}}+\frac{1}{\varepsilon}(g(\phi)-(1-\varepsilon) f(\phi)) .
\end{gathered}
$$

Thus $t(r, \phi)$ coincides with $f(\phi)$ and $g(\phi)$, resp., for $r=\varepsilon$ and $r=\varepsilon-\varepsilon^{2}$, resp.

The energy of $t(r, \phi)$ on the annulus $B\left(x_{0}, \varepsilon\right) \backslash B\left(x_{0}, \varepsilon-\varepsilon^{2}\right)$ is given by

$$
\begin{aligned}
E(t)= & \int_{r=\varepsilon-\varepsilon^{2}}^{\varepsilon} \int_{\phi=0}^{2 \pi}\left(\frac{1}{\varepsilon^{4}}|f(\phi)-g(\phi)|^{2}\right. \\
& \left.+\frac{1}{r^{2}}\left|\left(\frac{r}{\varepsilon^{2}}-\frac{1-\varepsilon}{\varepsilon}\right) f^{\prime}(\phi)+\left(\frac{1}{\varepsilon}-\frac{r}{\varepsilon^{2}}\right) g^{\prime}(\phi)\right|^{2}\right) r d r d \varphi .
\end{aligned}
$$

Using (10), $\left|f^{\prime}(\phi)\right|=O(\varepsilon)$ and $\left|g^{\prime}(\phi)\right|=O(\varepsilon)$ we calculate $E(t)=O(\varepsilon)^{3}$, and hence also,

$$
E\left(\pi^{-1} \circ t\right)=O\left(\varepsilon^{3}\right)
$$

We put $v=\pi^{-1} \circ t$ on the annulus $B\left(x_{0}, \varepsilon\right) \backslash B\left(x_{0}, \varepsilon-\varepsilon^{2}\right)$. Therefore

$$
\begin{aligned}
E(v)= & E\left(\tilde{u} \mid \Sigma_{1} \backslash B\left(x_{0}, \varepsilon\right)\right)+E\left(\pi^{-1} \circ w \mid B\left(x_{0}, \varepsilon-\varepsilon^{2}\right)\right) \\
& +E\left(\pi^{-1} \circ t \mid B\left(x_{0}, \varepsilon\right) \backslash B\left(x_{0}, \varepsilon-\varepsilon^{2}\right)\right) \\
= & E(\tilde{u})-O\left(\varepsilon^{2}\right)+\operatorname{Area}\left(\Sigma_{2}\right)+O\left(\varepsilon^{3}\right),
\end{aligned}
$$

since $E\left(\tilde{u} \mid B\left(x_{0}, \varepsilon\right)\right)=O\left(\varepsilon^{2}\right)$, because $d \tilde{u}\left(x_{0}\right) \neq 0$, and the energy of $\pi^{-1} \circ w$ is the area of its image, since $\pi$ and $w$ and hence also $\pi^{-1} \circ w$ are conformal. Thus, for sufficiently small $\varepsilon \geqslant 0$ (9) is satisfied, and the proof is complete.

Remark. In spite of the argument presented in $\$ 1$, our proof shows that in many cases, we even get more than two homotopically distinct harmonic maps. 
Apart from the possibility that there might be several homotopy classes $\tilde{\alpha}$ with $E_{\tilde{\alpha}}=E$, our construction of the map $v$ can yield two different new homotopy classes as soon as the functional determinant of $\tilde{u}$, a map with $E(\tilde{u})=E$, changes sign or if there exists a point where the functional determinant of $\tilde{u}$ vanishes, while $d \tilde{u}$ is nonzero at this point.

\section{References}

[1] H. Brézis \& J. M. Coron, Multiple solutions of H-systems and Rellich's conjecture, Comm. Pure Appl. Math., to appear.

[2] L Large solutions for harmonic maps in two dimensions, Comm. Math. Phys., to appear.

[3] J. Eells \& J. C. Wood, Restrictions on harmonic maps of surfaces, Topology 15 (1976) 263-266.

[4] H. Federer, Geometric measure theory, Die Grundlehren der Math. Wiss., No. 153, Springer, New York, 1969.

[5] S. Hildebrandt, H. Kaul \& K. O. Widman, An existence theorem for harmonic mappings of Riemannian manifolds, Acta Math. 138 (1977) 1-16.

[6] J. Jost, Existence proofs for harmonic maps with the help of a maximum principle, Math. Z., 184 (1983), 489-496.

[7] __ Harmonic maps between surfaces, Lecture Notes in Math., Vol. 1062, Springer, Berlin, 1984.

[8] L. Lemaire, Applications harmoniques de surfaces Riemanniennes, J. Differential Geometry 13 (1978) 51-78.

[9] __ Boundary value problems for harmonic and minimal surfaces into manifolds, Ann. Scuola Norm. Sup. Pisa Cl. Sci. (4) 9 (1982) 91-103.

[10] J. Sacks \& K. Uhlenbeck, The existence of minimal immersions of 2-spheres, Ann. of Math. (2) 113 (1981) 1-24.

[11] R. Schoen \& S. T. Yau, Existence of incompressible minimal surfaces and the topology of three dimensional manifolds with non-negative scalar curvature, Ann. of Math. (2) 110 (1979) 127-142.

[12] K. Steffen, On the nonuniqueness of surfaces with prescribed constant mean curvature spanning a given contour, Arch. Rational Mech. Anal., to appear.

[13] M. Struwe, Nonuniqueness in the Plateau problem for surfaces of constant mean curvature, Arch. Rational Mech. Anal., to appear.

[14] H. Wente, A general existence theorem for surfaces of constant mean curvature, Math. Z. 120 (1971) 277-288. 
